\title{
Absinth gegen Morbus Crohn
}

\begin{abstract}
Der Tumor-Nekrose-Faktor $\alpha$ (TNF- $\alpha$ ) scheint nach neueren Erkenntnissen beim Morbus Crohn eine Schlüsselrolle zu spielen. Mit einem Absinthextrakt lässt sich die Bildung von TNF- $\alpha$ in vitro hemmen. Eine Studie sollte zeigen, ob sich der Effekt auch in vivo nutzen lässt.
\end{abstract}

- 20 Patienten, die an Morbus Crohn litten, wurden in zwei Gruppen randomisiert. Die Experimentalgruppe $(\mathrm{n}=10)$ erhielt sechs Wochen lang zusätzlich zur Standardtherapie täglich $3 \times 750$ mg eines Absinthextrakts (Seda-
Crohn $\left.{ }^{\circledR}\right)$. Die Kontrollgruppe wurde nur mit der Standardtherapie behandelt.

Die Ergebnisse zeigen, dass unter der Absinththerapie die TNF- $\alpha$-Spiegel signifikant deutlicher abfielen. Klinisch wurden in der Absinthgruppe acht Remissionen beobachtet, während nur zwei Patienten in der Kontrollgruppe Remissionen berichteten. Die Autoren meinen, dass diese Untersuchung eine Basis schafft, den Absinthextrakt in Situationen zu testen, in denen proinflammatorische Zytokine eine Rolle spielen.

\section{Kommentar}

Da die Studie eindeutig ein Pilotprojekt ist, kann man die kleine Fallzahl vielleicht entschuldigen. Nur schwer zu verstehen ist jedoch der Umstand, dass in der Kontrollgruppe kein Placebo gegeben wurde. Mit relativ geringem Mehraufwand wären die Daten wesentlich überzeugender gewesen.

E. ERNST =

- S. Krebs S, T. N. Omer, B. Omer

Wormwood (Artemisia absinthium) suppresses tumour necrosis factor alpha and accelerates healing in patients with Crohn's disease - A controlled clinical trial. Phytomedicine 17 (2010) 305-309

\section{Woran denken Sie bei Schluckauf, Übelkeit und Erbrechen?}

Ein 15-jähriges Mädchen litt seit zwei Wochen unter unstillbarem Schluckauf, Übelkeit und Erbrechen. Die Symptomatik hatte eines Morgens unmittelbar nach dem Aufwachen begonnen.

— Die körperliche Untersuchung und sämtliche Labortest waren bis auf eine erhöhte Serumamylase unauffällig. Es wurde deshalb ein MRT des Abdomens durchgeführt, das aber ebenfalls ohne Befund blieb. Beim MRT des Schädels zeigte sich aber eine signalintensive Läsion in der Medulla, unter Einbeziehung der Area postrema (Abb. A). Der
Liquorbefund wie auch die visuell evozierten Potenziale waren unauffällig. Allerdings fanden sich im Serum Antikörper gegen Aquaporin-4.

Im Lauf der dreiwöchigen stationären Behandlung entwickelte die $\mathrm{Pa}$ tientin eine internukleäre Ophthalmoplegie. Das wiederholte MRT des Kopfes zeigte eine Zunahme der Größe der Läsion in der Medulla oblongata (Abb. B) und eine zweite hyperintense Läsion im Thalamus.

Man stellte die Diagnose einer Neuromyelitis optica und begann eine Therapie mit $1000 \mathrm{mg}$ Methylprednisolon täglich. Bereits nach dem ersten Be-

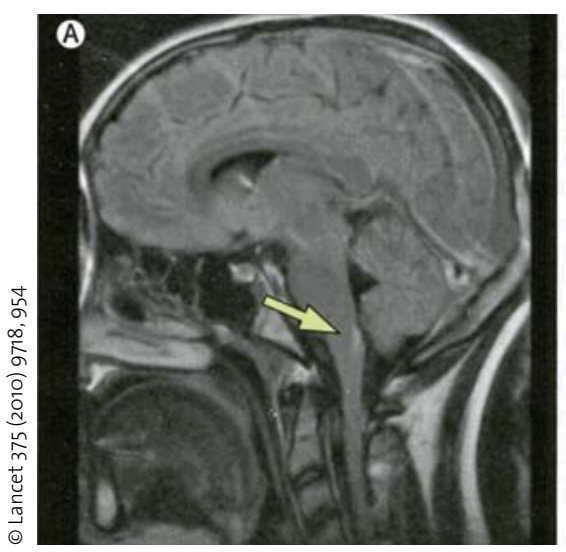

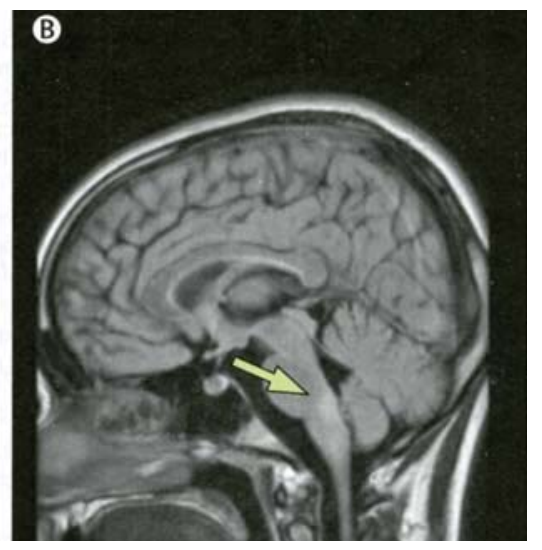

handlungstag verschwand die internukleäre Ophthalmoplegie und nach fünf Tagen war das Erbrechen verschwunden.

\section{Kommentar}

Die Neuromyelitis optica ist eine entzündliche Erkrankung, die Rückenmark und Nervus opticus befallen kann. Typischerweise ist der Verlauf intermittierend, das Gehirn bleibt ausgespart. Als Erstes dachte man bei der Symptomatik natürlich an eine multiple Sklerose, doch konnte diese Diagnose durch die Bildgebung und den Liquorbefund ausgeschlossen werden. Auch wenn die Neuromyelitis optica ein sehr seltenes Krankheitsbild ist, ist die Symptomenkombination aus unstillbarem Schluckauf in Kombination mit Übelkeit und Erbrechen doch so charakteristisch, dass man als Arzt diese Diagnose nicht ganz vergessen sollte.

H. S. FÜEßL =

\footnotetext{
- J. Riphagen et al.

(Korr.: Joanne Riphagen, MD, Department of Pediatric Neurology, Canisius Wilhelmina Hospital, PO Box 9015,6500 GS Nijmegen, Netherlands, e-mail: j.riphagen@cwz.nl): Hiccups, nausea, and vomiting: water channels under attack! Lancet 375 (2010) 9718, 954
}

A: Läsion in der Medulla (Pfeil); B: Größenzunahme nach drei Wochen. 